\title{
Evaluation of the effects of four media on human intestinal microbiota culture in vitro
}

\author{
Fu Yousi ${ }^{1}$, Chen Kainan ${ }^{1}$, Zhang Junnan ${ }^{1}$, Xiao Chuanxing ${ }^{2}$, Fan Lina ${ }^{2}$, Zhang Bangzhou ${ }^{2}$, Ren Jianlin²* \\ and Fang Baishan ${ }^{1 *}$
}

\begin{abstract}
The human intestinal microbiota has an important role in the maintenance of human health and disease pathogenesis. The aim of this research was to investigate the impact of four media on human intestinal microbiota metabolite and composition changes, we performed in vitro batch culture using intestinal microbiota samples from three fecal microbiota transplantation (FMT) donors. After $48 \mathrm{~h}$ culture, gut microbiota medium (GMM) had the highest production of acetic acid $(73.00 \pm 7.56 \mathrm{mM})$ and propionic acid $(16.79 \pm 1.59 \mathrm{mM})$, bacterial growth media $(B G M)$ had the highest production of butyric acid $(13.39 \pm 0.56 \mathrm{mM})$. In addition, brain heart infusion (BHI) promoted $(\mathrm{p}<0.05)$ the growth of Bacteroidetes, especially Bacteroides after $48 \mathrm{~h}$, GMM resulted in a significant increase $(p<0.05)$ in Actinobacteria and increased the beneficial genus Bifidobacterium, fastidious anaerobe broth (FAB) increased Firmicutes population, and BGM promoted the growth of Escherichia-Shigella and Akkermansia. The results suggest that four media had different effects on the human intestinal microbiota metabolism and composition in vitro. These results may facilitate the culture of bacteria from the human intestinal microbiota.
\end{abstract}

Keywords: Intestinal microbiota, Short chain fatty acid, In vitro culture, 16S rRNA

\section{Introduction}

The human gastrointestinal tract harbors at least $10^{14}$ bacterial cells of 400-1000 bacterial species to form the intestinal microbiota that has an important role in the maintenance of human health (Eckburg et al. 2005; Tremaroli and Bäckhed 2012). Interactions of the human microbiota with the host are usually mediated by bacterial metabolic products such as vitamins, amino acids and short chain fatty acid (SCFA) (Tramontano et al. 2018). Changes in the equilibrium of the gut microbial ecosystem have been associated with a range of diseases, including obesity, type II diabetes and inflammatory bowel disease (Makki et al. 2018; Soto et al. 2018). Fecal microbiota transplantation (FMT) is a new method to treat a variety of dysbiosis-associated gut diseases, such as Clostridium difficile infection (CDI) (Surawicz et al.

\footnotetext{
*Correspondence: jianlin.ren@126.com; fbs@xmu.edu.cn

${ }^{1}$ Department of Chemical and Biochemical Engineering, College

of Chemistry and Chemical Engineering, Xiamen University, No. 422, Siming South Road, Xiamen 361005, Fujian, China

${ }^{2}$ Zhongshan Hospital Department of Gastroenterology, Xiamen University, No. 201-209, Hubinnan Road, Xiamen 361005, Fujian, China
}

2013) and chronic hepatitis B (Ren et al. 2017). FMT involves transfer of intestinal microbiota from healthy donors to patients to correct intestinal microbiota dysbiosis (Staley et al. 2018). The intestinal microbiota from FMT donors can be used to represent the healthy human intestinal microbiota (Gupta and Khanna 2017).

Culturomics is a culturing approach that uses multiple culture conditions, 16S rDNA sequencing and MALDITOF mass spectrometry for the identification of bacterial species (Lagier et al. 2018). The first step of culturomics is to diversify the intestinal microbiota sample into different culture media, promoting the growth of fastidious bacteria from the human gut; the next step is to optimize culture conditions to promote the growth of fastidious microorganisms at lower concentrations (Lagier et al. 2018). Anaerobes are dominant members of the human intestinal microbiota (Pham and Mohajeri 2018). In addition, culturing obligate anaerobes requires oxygen-free environment and complex media with many supplements (macroelements and growth factors) (Lau et al. 2016). In vitro batch culture is a semi-representative model and provides a time- and cost-effective way to culture human 
intestinal microbiota under simulated physiological conditions (anaerobiosis, culture medium, $37{ }^{\circ} \mathrm{C}$ ) (Williams et al. 2015). The advantages of in vitro batch culture are fast, cheap, easy to operate and reproducible (Pham and Mohajeri 2018), and this approach can serve as highthroughput initial investigations of the culturomics by providing important clues to guide further studies.

Therefore, the objective of the present study was to investigate the effects of four culture media on human intestinal microbiota from FMT donors using an in vitro batch culture model. We envision that these results can improve our knowledge of human microbial ecosystem and facilitate human intestinal microbiota culture in vitro.

\section{Materials and methods Materials}

High purity SCFA standards for gas chromatography (GC) analysis, including acetic acid, propionic acid and butyric acid, were purchased from Sigma Aldrich Chemical Co. (St Louis, MO, USA). L-Cysteine hydrochloride monohydrate, bile salts, tryptone, yeast extract, glucose and other chemicals were obtained from Sangon Biotech (Shanghai, China). Media used in this study included: brain heart infusion (BHI) media (Solarbio, Beijing, China), gut microbiota medium (GMM) without agar (Goodman et al. 2011), fastidious anaerobe broth (FAB, Solarbio, Beijing, China), bacterial growth media (BGM) (Mcdonald et al. 2013). All media were prepared following the manufacturer's instructions.

\section{Preparation of human intestinal microbiota}

Three human intestinal microbiota samples were collected at Zhongshan Hospital of Xiamen University from healthy FMT donors who had not received antibiotics within 3 months of sampling, and had no history of digestive disease (Ren et al. 2017). Intestinal microbiota samples from three donors were pooled after collection. The study was approved by Xiamen University and Zhongshan Hospital of Xiamen University, China. All FMT donors provided written informed consent. In vitro culture was performed in compliance with the relevant laws and institutional guidelines.

\section{In vitro culture}

In vitro culture was performed in $150 \mathrm{~mL}$ volumes of sterilized culture medium in $250 \mathrm{~mL}$ containers. Sterilized culture medium was added to the vessels, which were then maintained at $37{ }^{\circ} \mathrm{C}$ with magnetic stirring at $150 \mathrm{rpm}$. Prior to culture, the vessels were sparged with $\mathrm{N}_{2}$ gas to obtain an anaerobic environment. The following day, $15 \mathrm{~mL}$ of fecal inoculum were inoculated into each container. Batch culture was carried out anaerobically for
$48 \mathrm{~h}$ at $37^{\circ} \mathrm{C}$, with samples collected at seven time points $(0,4,8,12,24,36$ and $48 \mathrm{~h})$ over the course of the experimental period. Samples were stored at $-80{ }^{\circ} \mathrm{C}$ until further analysis.

\section{Determination of SCFA content}

For SCFA analysis, samples were filtered through a $0.22 \mu \mathrm{m}$ membrane prior to analysis. The SCFA content was analyzed using an Agilent 7890A gas chromatograph with flame ionization detector. A gas chromatography column (AT-FFAP, 1116-342, $30 \mathrm{~m} \times 0.32 \mathrm{~mm}$ i.d. ATEO, Lanzhou, China) coated with a $0.25 \mu \mathrm{m}$ thickness film was used for the analysis. Chromatographic analysis was performed according to the manufacturer's instructions. The initial oven temperature was $80^{\circ} \mathrm{C}$ for 5 min, which was raised to $250{ }^{\circ} \mathrm{C}$ at $5{ }^{\circ} \mathrm{C}$ per min. The temperatures of the injection port and the flame ionization detector were $250{ }^{\circ} \mathrm{C}$ and $260{ }^{\circ} \mathrm{C}$, respectively. The flow rate of the carrier gas, nitrogen, was set at $19.0 \mathrm{~mL} / \mathrm{min}$, with a split ratio of 1:10. The flow rates of hydrogen and air were $30 \mathrm{~mL} / \mathrm{min}$ and $300 \mathrm{~mL} / \mathrm{min}$, respectively. The injection volume was $1 \mu \mathrm{L}$. Each sample was examined three times independently.

\section{DNA extraction and 16S rRNA gene sequencing}

Samples $(1.5 \mathrm{~mL}$ each) collected from culture vessels were centrifuged at $13,000 \times g$ for $10 \mathrm{~min}$, the supernatant discarded, and the pellets were resuspended in 750 $\mu \mathrm{L}$ of bead solution. DNA extraction was then carried out using a Power Fecal DNA Isolation Kit (12830-50; MOBIO Laboratories, Carlsbad, CA, USA) according to manufacturer's instructions. Primers F341 (5'-CCTAYG GGRBGCASCAG-3') and R806 (5'-GGACTACNNGGG TATCTAAT- $3^{\prime}$ ) were then used to amplify the V3-V4 domain of the bacterial $16 \mathrm{~S}$ rRNA gene. PCR reactions contained 100-300 ng of template, $10 \times$ buffer (Toyobo, Osaka, Japan), $2 \mathrm{mM}$ dNTPs, $25 \mathrm{mM} \mathrm{MgSO}_{4}, 10 \mathrm{mM}$ forward and $10 \mu \mathrm{M}$ reverse primers, $1 \mu \mathrm{L}$ of KOD enzyme, and sterile double-distilled $\mathrm{H}_{2} \mathrm{O}$ in a final volume of 50 $\mu \mathrm{L}$. Reaction conditions consisted of an initial denaturation at $94{ }^{\circ} \mathrm{C}$ for $2 \mathrm{~min}$, followed by $28-35$ cycles of $98^{\circ} \mathrm{C}$ for $10 \mathrm{~s}, 62-66^{\circ} \mathrm{C}$ for $30 \mathrm{~s}$, and $68^{\circ} \mathrm{C}$ for $30 \mathrm{~s}$, and a final extension of $68^{\circ} \mathrm{C}$ for $5 \mathrm{~min}$. The resulting amplicons were purified using AMPure XP beads (Beckman Coulter, Brea, CA, USA). Purified libraries were sequenced using the Illumina Hiseq 2500 platform (Illumina, San Diego, CA, USA). Raw Illumina read data for all samples were deposited in the NCBI Sequence Read Archive database under accession number PRJNA506872.

\section{Community structure analysis}

Analysis of raw Illumina fastq files was performed using quantitative insights into microbial ecology 2 (Qiime 2, 


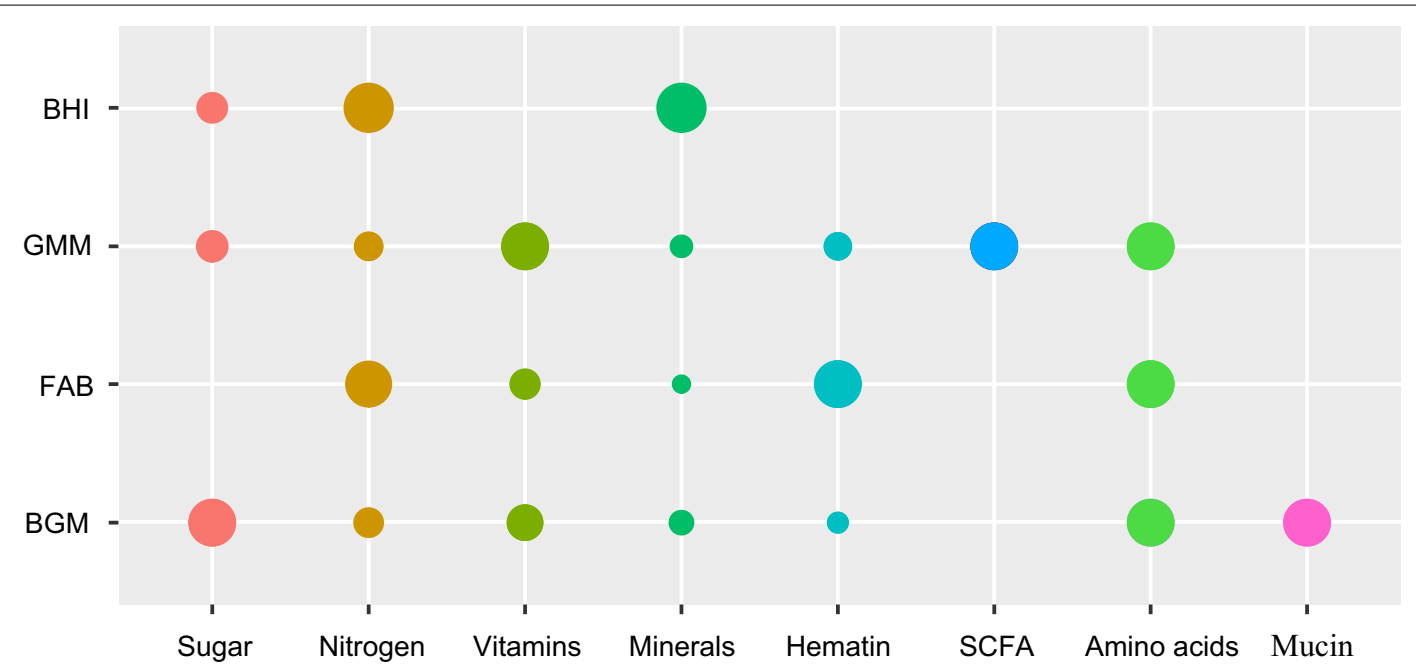

Fig. 1 Comparison of nutrient group representation across four media. Circle size correlates linearly with the quantity of the respective nutrient group

ver. 2018.2 https://qiime2.org/). Sequences were quality filtered, trimmed, de-noised, and merged using DADA 2 (Callahan et al. 2016). Chimeric sequences were then identified and removed, taxonomy was classified using the Silva 119 16S rRNA database (Quast et al. 2013). To analyze the alpha diversity, Shannon index and observed OTUs were calculated by QIIME 2. For the beta diversity analysis, constrained principal coordinates analysis (CPCoA) of Bray-Curtis distances constrained by group was computed using the capscale function implemented in the vegan $\mathrm{R}$ library (version $2.5-2$ ). In order to mine deeper data of microbial diversity of the difference among the four groups, significance test was conducted with linear discriminant analysis effect size (LEfSe, http://hutte nhower.sph.harvard.edu/galaxy). The network was generated using the CoNet plugin (version 1.1.1) for Cytoscape (version 3.6.1) on the basis of the nonparametric Spearman correlation coefficients, with a minimal cutoff threshold rho of $0.6(\mathrm{p}<0.05$, Benjamini-Hochberg corrected) (Yan et al. 2018).

\section{Statistical analysis}

Data were expressed as mean \pm standard deviation (SD). All of the four groups were conducted with twelve same intestinal microbiota samples. Statistical comparisons of SCFA production and alpha diversity data were evaluated by one-way analysis of variance, and compared using the Tukey test at $5 \%$ confidence level using SPSS software version 22.0 (SPSS, Chicago, IL, USA). Values of $\mathrm{p}<0.05$ were considered statistically significant. Venn diagrams and constrained principal-coordinate analysis (CPCoA) were created in the $\mathrm{R}$ software (version 3.5.0).

\section{Results}

\section{Comparison of media composition}

In this study, the human intestinal microbiota from FMT donors was cultured in four types of media including BHI, GMM, FAB and BGM. All media were composed of commercially available components (Fig. 1). BHI media consisted of sugar, nitrogen and the highest concentration of minerals in comparison with other three media. GMM media contained SCFA and the highest concentration of vitamins among four media. FAB media did not contain sugar components and BGM media contained mucin and the highest concentration of sugar among four media.

\section{SCFA production and $\mathrm{pH}$ shift}

Anaerobic cultures were conducted using the four types of media in the presence of human intestinal microbiota at initial $\mathrm{pH}$ values of 7.0. The production (minus the concentration of SCFA at $0 \mathrm{~h}$ ) of SCFA, including acetic, propionic and butyric acid is presented in Fig. 2. Acetic acid production from 4 to $48 \mathrm{~h}$ was similar for the BHI, FAB and BGM groups. The GMM group produced $73.00 \pm 7.56 \mathrm{mM}$ acetic acid after $48 \mathrm{~h}$, which was significantly $(\mathrm{p}<0.05)$ higher than that in the FAB group. Similarly, the GMM group produced the highest level of propionic acid $(16.79 \pm 1.59 \mathrm{mM})$, which was significantly $(p<0.05)$ higher than that in the FAB group. The highest production of butyric acid $(13.39 \pm 0.56 \mathrm{mM})$ was obtained in the BGM group, and significantly $(\mathrm{p}<0.05)$ more than the FAB group. After $48 \mathrm{~h}$, the production of total SCFA was significantly $(\mathrm{p}<0.05)$ higher in the GMM group compared to that in the FAB groups. The $\mathrm{pH}$ shift as a reflection of culture was evaluated (Fig. 2E). In the 


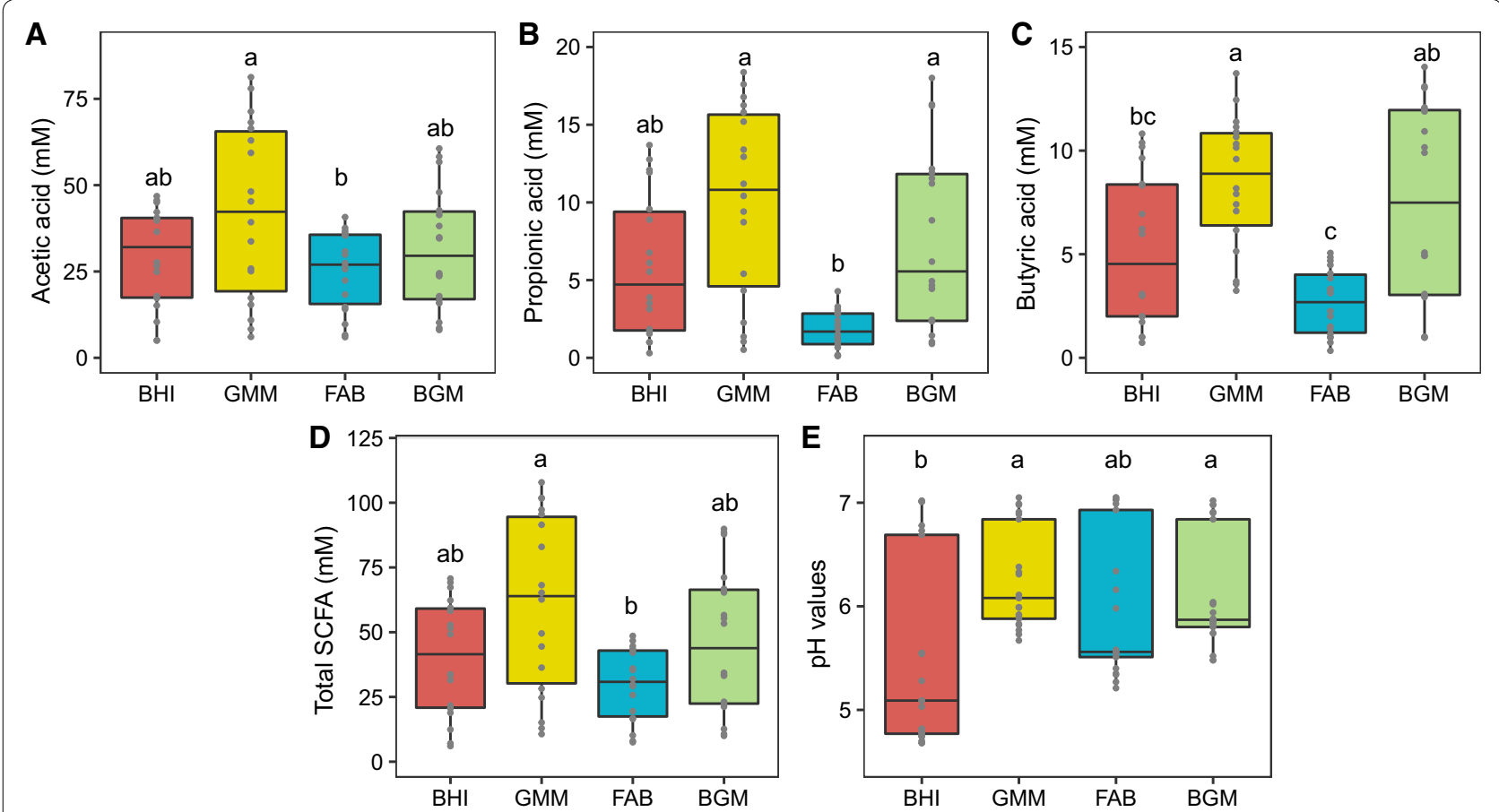

Fig. 2 SCFA production from 4 to $48 \mathrm{~h}$ and $\mathrm{pH}$ values from 0 to $48 \mathrm{~h}$ of $\mathrm{BH}, \mathrm{GMM}, \mathrm{FAB}$ and $\mathrm{BGM}$ groups. A Acetic acid, B propionic acid, $\mathbf{C}$ butyric acid, $\mathbf{D}$ total SCFA, E pH values. Data were analyzed using ANOVA with Tukey HSD $(p<0.05)$

BHI group, the $\mathrm{pH}$ value decreased to $4.80 \pm 0.02$ after $48 \mathrm{~h}$, which was significantly $(\mathrm{p}<0.05)$ lower than that in the GMM and BGM groups. Furthermore, there were no significant $(\mathrm{p}>0.05)$ differences in $\mathrm{pH}$ values among the GMM, FAB and BGM groups from $0 \mathrm{~h}$ to $48 \mathrm{~h}$.

\section{Composition of microbial community}

The intestinal microbial community structures in four groups were further investigated by high-throughput sequencing. Two alpha diversity measures were calculated including Shannon index and observed OTUs (Fig. 3A, B). For this, the Shannon index of the BHI group was significantly $(p<0.05)$ higher than that of other three groups (Fig. 3A). Regarding the comparison of community richness, the BHI group had a significantly $(\mathrm{p}<0.05)$ higher number of observed OTUs than other three groups (Fig. 3B). The Venn diagram showing overlap in the observed OTUs among the samples revealed that there were 137 shared OTUs in the BHI group (Fig. 3C), 96 in the GMM group (Fig. 3D), 116 in the FAB group (Fig. 3E) and 90 in the BGM group (Fig. 3F) from 0 to $48 \mathrm{~h}$ culture. A higher number of OTUs were retained in the BHI group compared to other three groups after $48 \mathrm{~h}$ culture. The analysis of constrained principal-component $(\mathrm{CPCoA})$ indicated that there was a significant separation of the four groups, especially between the BHI and GMM groups from 4 to $48 \mathrm{~h}$ culture (Fig. 4). This analysis revealed that the bacteria communities differences between the BHI and GMM were larger than the differences between the FAB and BGM groups.

The relative abundance of the top 4 most abundant intestinal microbiota at the phylum-level in the BHI, GMM, FAB and BGM groups is illustrated by histograms in Fig. 5. The relative abundance of the Actinobacteria phylum in the GMM group was significantly $(\mathrm{p}<0.05)$ higher than BHI, FAB and BGM groups. The relative abundance of Bacteroidetes in the four groups from 4 to $48 \mathrm{~h}$ culture was decreased compared to $0 \mathrm{~h}$ (FMT donors intestinal microbiota samples), and Bacteroidetes of BHI group was significantly $(\mathrm{p}<0.05)$ higher than that of other three groups. A significant increase of Firmicutes was observed in the FAB group when compared to other three groups from 4 to $48 \mathrm{~h}$ culture. Compared to $0 \mathrm{~h}$, there was a notable increasing relative abundance of Proteobacteria in the four groups from 4 to $48 \mathrm{~h}$, and Proteobacteria of BGM group was significantly $(\mathrm{p}<0.05)$ higher than that of other groups.

At the genus level (Fig. 6), a significant increase of Clostridium, Enterococcus, Escherichia-Shigella and Streptococcus and a significant decrease of Faecalibacterium, Megamonas, Prevotella and Ruminococcaceae_un were observed in the four groups from 4 to $48 \mathrm{~h}$ when compared to $0 \mathrm{~h}(\mathrm{p}<0.05)$. During the period from 4 to $48 \mathrm{~h}$ culture, the relative abundance of Bacteroides and 

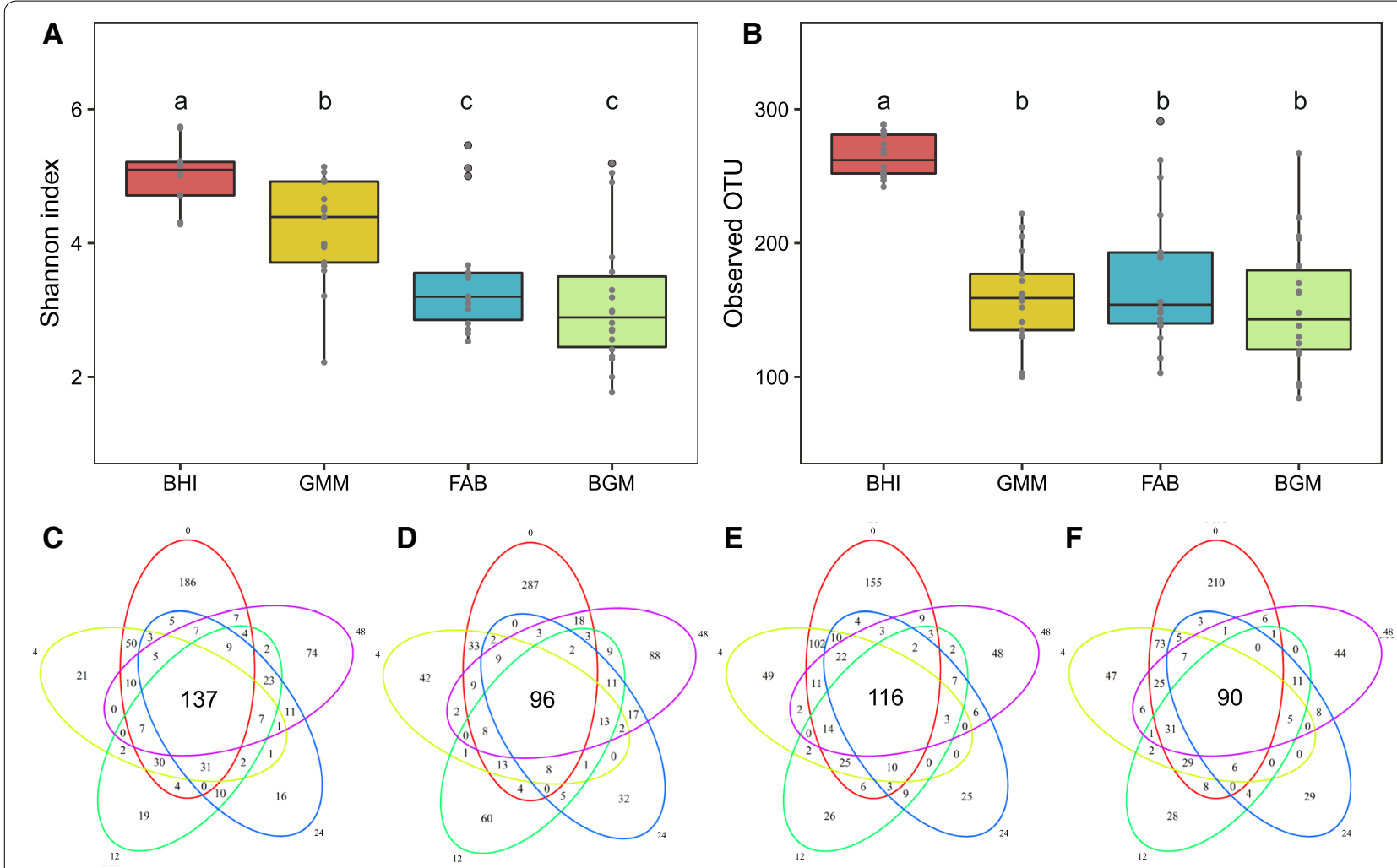

Fig. 3 Community diversity as measured by the Shannon index (A) and Observed OTUs (B) of the BHI, GMM, FAB and BGM groups from 4 to $48 \mathrm{~h}$ culture. Venn diagrams illustrating the number of unique and shared OTUs among BHI (C), GMM (D), FAB (E) and BGM (F) groups at $0,4,12,24,48 \mathrm{~h}$

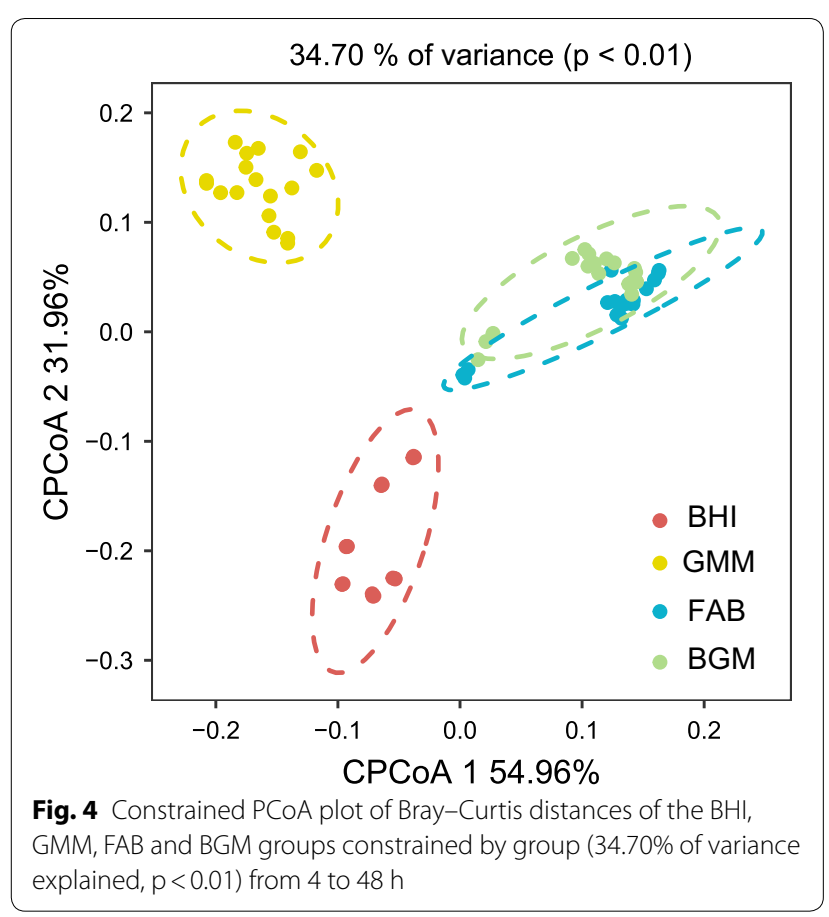

Fig. 4 Constrained PCoA plot of Bray-Curtis distances of the BHI, explained, $\mathrm{p}<0.01$ ) from 4 to $48 \mathrm{~h}$
Lachnospira in the BHI group was higher than that in other groups; the amounts of Bifidobacterium in GMM group ranging from 0.01 to $0.81 \%$ were significantly $(\mathrm{p}<0.05)$ higher than other groups; the relative abundance of Citrobacter and Coprococcus in the GMM group was also higher than other groups $(\mathrm{p}<0.05)$; there was a notable increasing relative abundance of Clostridium and Enterococcus in the FAB group compared to other three groups $(\mathrm{p}<0.05)$; the BGM group indicated the highest relative abundance of Akkermansia and EscherichiaShigella among the four groups. In addition, the relative abundance of Faecalibacterium in the four groups was similar from 4 to $48 \mathrm{~h}(\mathrm{p}>0.05)$.

Additionally, bacterial taxa with significant difference among the BHI, GMM, FAB and BGM groups was demonstrated using LEfSe analysis (Fig. 7). LEfSe analysis indicated significant distinguishing bacteria with $\mathrm{Bac}$ teroides, Streptococcus and Klebsiella in the BHI group, with Citrobacter, Prevotella, Coprococcus, Bifidobacterium and Megamonas in the GMM group, with Clostridiaceae, Enterococcus and Butyricimonas in the FAB group, and with Escherichia-Shigella, Christensenellaceae, Oscillibacter and Akkermansia in the BGM group when compared with the other three groups. 

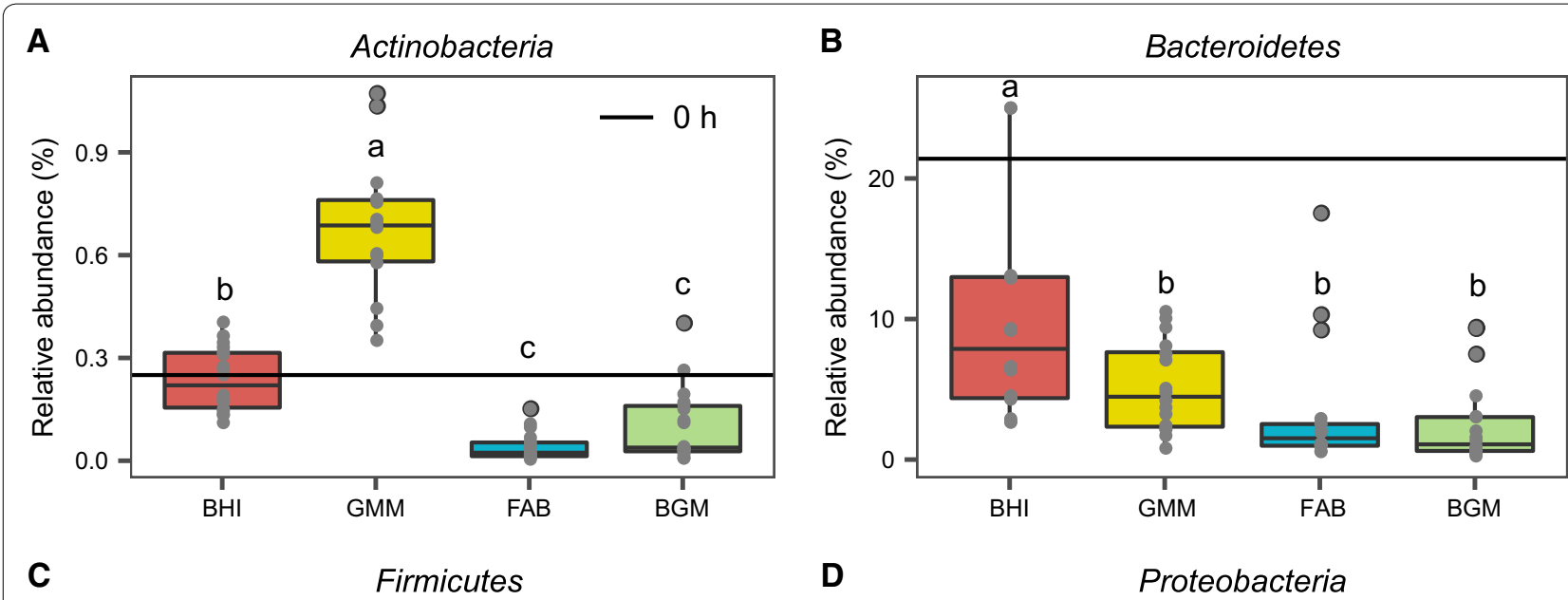

D

Proteobacteria
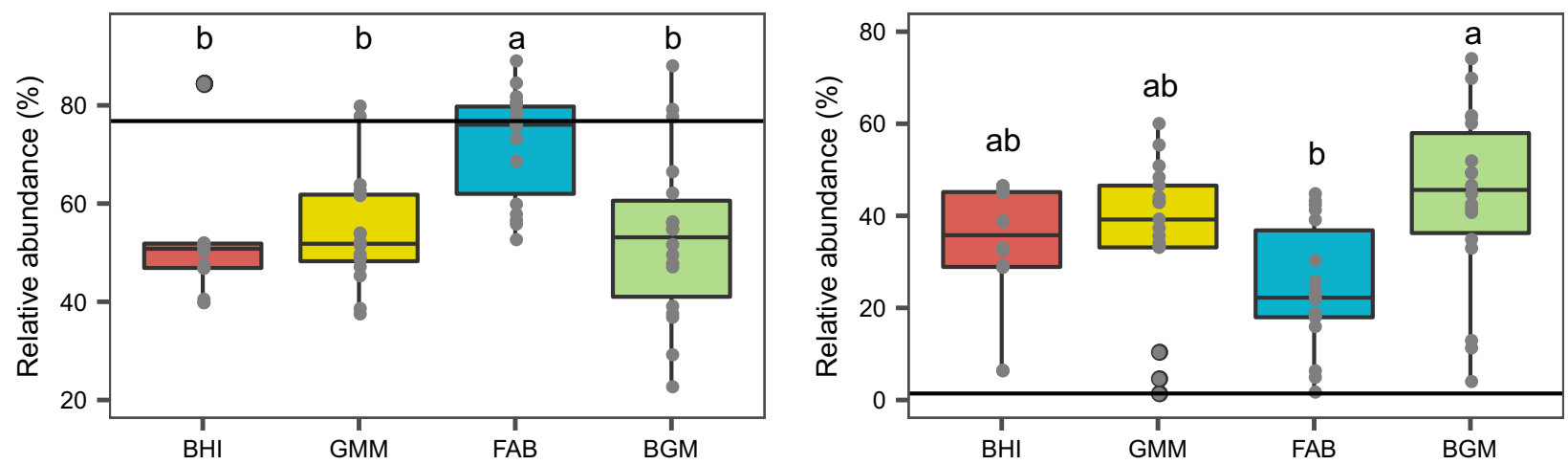

Fig. 5 The relative abundance of the top 4 most abundant intestinal microbiota at the phylum-level in $B H I, G M M, F A B$ and $B G M$ groups from 0 to 48 h. A Actinobacteria, B Bacteroidetes, C Firmicutes, D Proteobacteria. Data were analyzed using ANOVA with Tukey HSD $(p<0.05)$

To study the possible interactions among intestinal microbiota in the four groups, the relative abundance of genus higher than $0.1 \%$ were used to construct a network. As shown in Fig. 8, each node represents a bacterial genus and edges indicate significant cooccurrence (green) or mutual exclusion (red) interactions. Both significant co-occurrence and mutual exclusion (Spearman's rho $>0.6$ ) were observed in the four groups, confirming the presence of an interactive network of microorganisms. The network of the BHI group included 48 nodes and 203 edges. In this network, Megamonas had 21 edges, which were the key genus in the BHI group. The network of GMM group consisted of 31 nodes and 75 edges, and Ruminococcus were the nodes of the highest edges. In the FAB group network, 40 nodes and 103 edges were found, and Pseudobutyrivibrio (15 edges) were considered to be potential key genus. The network of BGM group included 34 nodes and 107 edges, Ruminococcaceae_un and Oscillibacter had 15 edges, which were the core genus in the BGM group.

\section{Discussion}

The aim of this study was to investigate the impact of four media upon human intestinal microbiota metabolite and composition in anaerobic batch cultures inoculated with human intestinal microbiota samples from FMT donors. SCFA produce by intestinal microbiota leads to many beneficial health outcomes to the host. The higher SCFA concentration created a lower $\mathrm{pH}$ environment in gut which may inhibit pathogen colonization (Jin et al. 2018). Acetic acid is the main metabolite of most intestinal microbiota, and it is an important source of energy for tissues and the substrate of cholesterol synthesis (Wang et al. 2018). Propionic acid is also a very important SCFA in the intestinal tract, and it has been reported that propionic acid could impact glucose metabolism in intestinal epithelium (Zadehtahmasebi et al. 2016). After 48 h culture, the GMM group produced the highest concentration of acetic acid and propionic acid among four groups. This result suggested that GMM media may facilitate the production of acetic and propionic acid from intestinal microbiota. Among all SCFA, butyric acid is considered a source for the intestinal epithelial cells and is implicated 


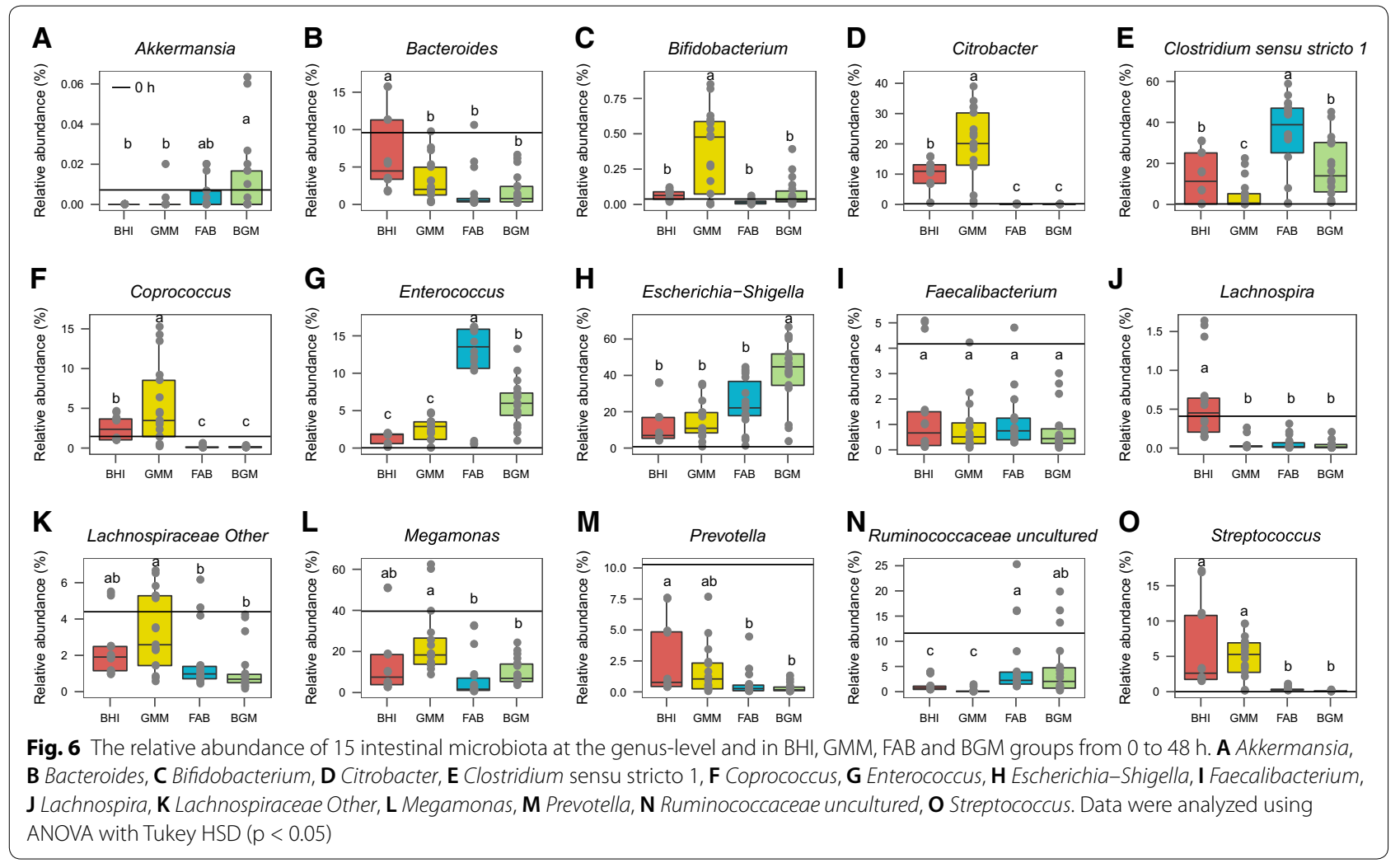

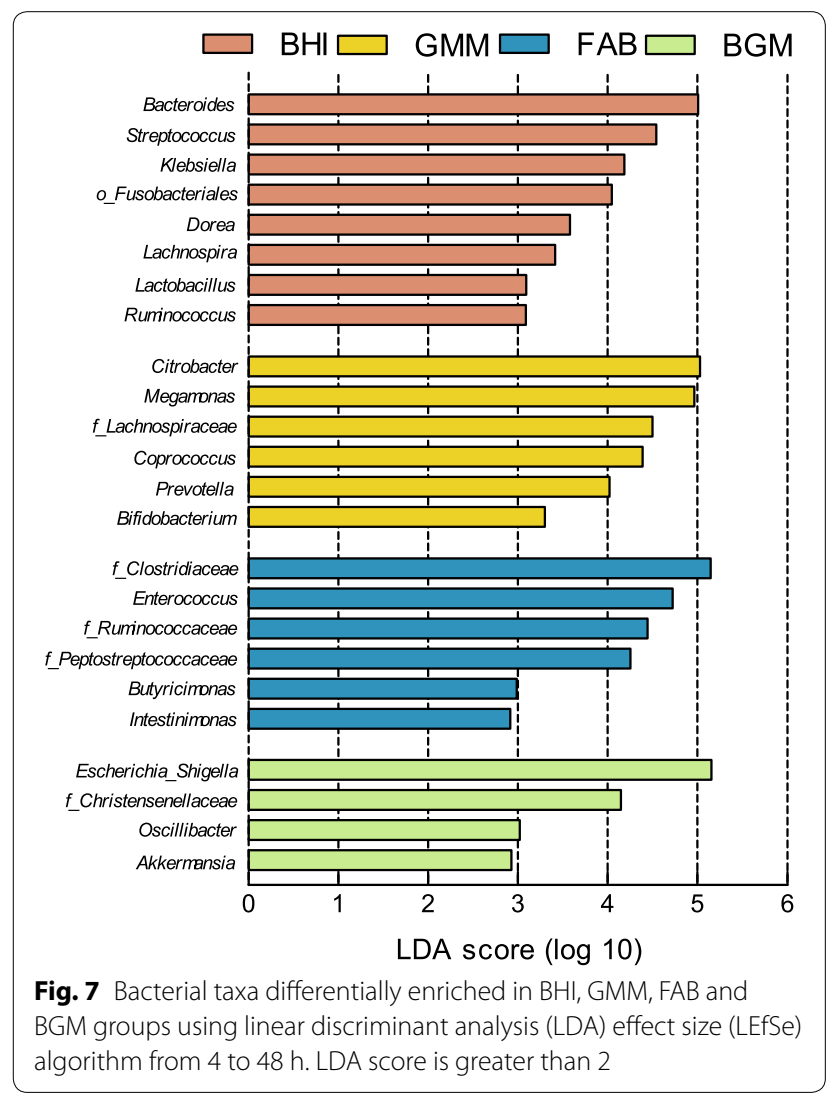

in reducing the incidence of colon inflammatory processes (Thomson et al. 2018). The media that promoted by far the highest production of butyric acid was BGM. This can be explained by the fact that the BGM media contains prebiotics like inulin. Similar in vitro studies with inulin have also shown this contributes to the highest average production of butyric acid (Carlson et al. 2017).

The profiles of microbial communities in the four groups indicated different patterns during $48 \mathrm{~h}$ culture. The alpha diversity is widely reported as an indicator of intestinal microbial communities state due to its relationship with productivity, functioning and stability (Reese and Dunn 2018). Compared with other three groups, intestinal microbial diversity, as estimated by Shannon index and observed OTUs, was significantly $(\mathrm{p}<0.05)$ higher in BHI group. We observed that a large amount of unique OTUs in the $0 \mathrm{~h}$ samples, indicating that those OTUs cannot be cultured during $48 \mathrm{~h}$ culture (Fig. 3CF). We speculate that differences between the in vivo and in vitro environments were the cause of OTUs lost. In vitro batch culture provides a way to culture human intestinal microbiota under simulated physiological conditions, while in vitro batch culture cannot mimic the interactions between microbiota and the host. Moreover, a higher number of OTUs were retained in the BHI 

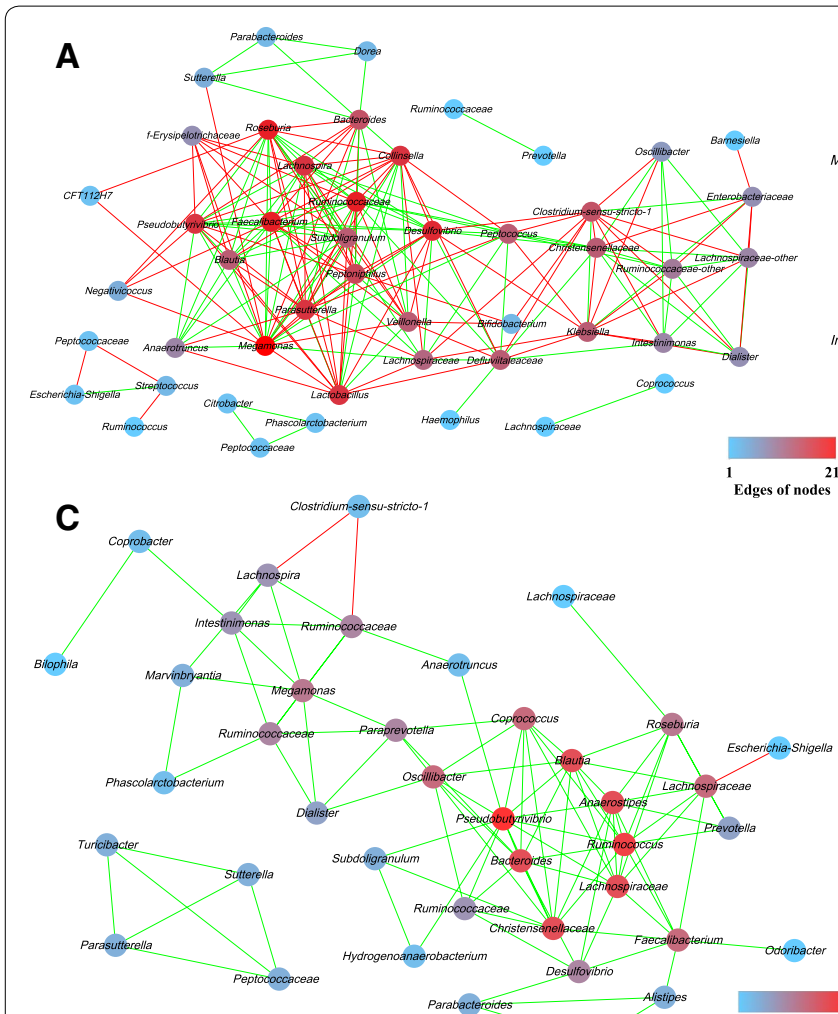

Butyricimonas
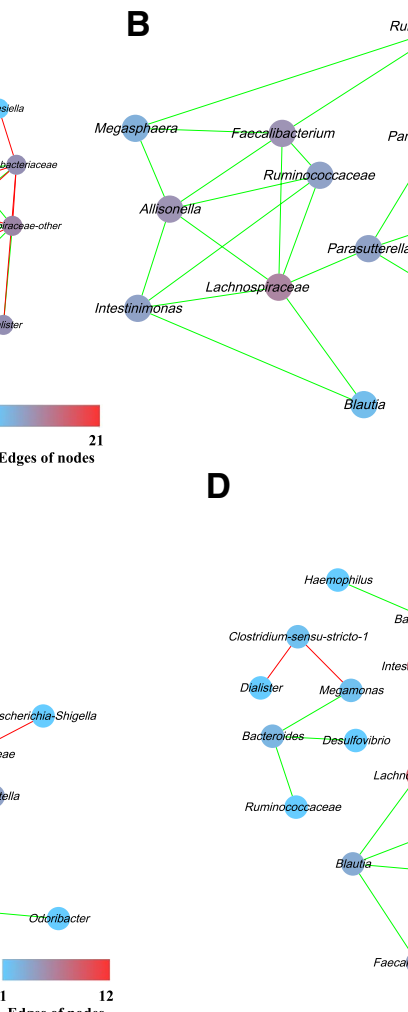

D

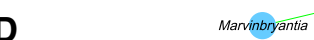

Marinbryantia

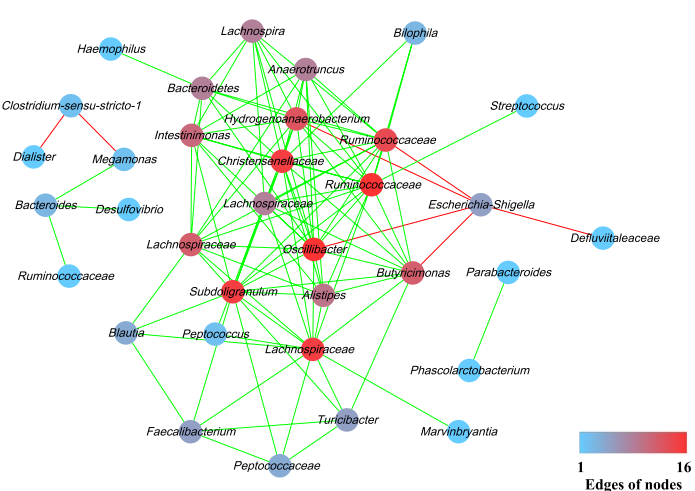

Fig. 8 Network analysis of the BHI (A), GMM (B), FAB (C) and BGM (D) samples indicating interactions among dominant bacteria from 4 to $48 \mathrm{~h}$. Nodes represent genus. The color of each node is proportional to the number of edges

group than other groups during the $48 \mathrm{~h}$ culture (Fig. 3). These results indicated that BHI media could maintain intestinal microbial diversity during in vitro culture.

At the genus level, Bacteroides, Lachnospira, Streptococcus and Klebsiella were the dominant bacteria in the BHI group in LEfse analysis. Bacteroides is one of the most abundant genera in the gut and has a positive correlation with propionic acid production (Nakano et al. 2013), and Lachnospira also plays an important role in SCFA production (Bang et al. 2018). Previous studies have reported that Streptococcus and Klebsiella were widely distributed in the human skin, mouth and intestine, and Streptococcus can degrade digested proteins into peptides in human gut (Macfarlane et al. 1988; Sekirov et al. 2010; Brisse et al. 2006). The relative abundance of Bifidobacterium, Prevotella, Citrobacter and Coprococcus in the GMM group was highest when compared to those in other groups. Bifidobacterium resides naturally in the gastrointestinal tract of healthy human adults and comprises a unique genus of bacteria, in which gas is not formed as an end product of metabolism (Routy et al. 2018). Bifidobacterium may exert multiple positive effects on human health ranging from prevention of obesity and infections to resolution of ulcerative colitis via the down regulation of TNF- $\alpha$ and IL-1 (Routy et al. 2018). Prevotella could improve human glucose metabolism and increase glycogen storage (Kovatcheva-Datchary et al. 2015). Citrobacter is the generally opportunistic pathogen and is among the leading causes of morbidity and mortality worldwide (Seo et al. 2017). The previous study found that the patients who suffered irritable bowel syndrome had minute Coprococcus (Kassinen et al. 2007). FAB group showed a significantly higher relative abundance of Clostridium sensu stricto and Enterococcus when compared to those in other three groups. Clostridium sensu stricto is usually regarded as the opportunistic pathogen (Zhou et al. 2018). The genus Enterococcus is a group of lactic acid bacteria, and they are sometimes associated with infections in humans (Fusco et al. 2017). Furthermore, the higher relative abundance of Akkermansia in the BGM group was observed in this study. Akkermansia is considered as next-generation probiotics for their anti-inflammatory properties, and is typically closely associated with the protective mucous lining of the human intestine (Schneeberger et al. 2015). 
Co-occurrence patterns identified using network analysis can reflect the interactions between microbes (Barberan et al. 2012). The network properties offer the possibility for quick and easy comparisons among complex data sets from different culture media to explore how the culture media influence the composition of microbial communities. Nodes that had high edges were often considered to be potential core nodes, if removed, would lead to large changes in the community (Faust et al. 2012). Thus, the results revealed that Megamonas, Ruminococcus, Pseudobutyrivibrio, and Ruminococcaceae_un and Oscillibacter were the key genera in the BHI, GMM, FAB and BGM groups, respectively.

Overall, the work presented here is one of few in vitro studies that compares the effects of culture media on the composition and metabolic activity of the human intestinal microbiota; the results showed the intestinal microbiota displayed different growth in the four media. We envision that these results can be used to facilitate human intestinal microbiota in vitro culture.

\begin{abstract}
Abbreviations
SCFA: short chain fatty acid; FMT: fecal microbiota transplantation; BHI: brain heart infusion; GMM: gut microbiota medium; FAB: fastidious anaerobe broth; BGM: bacterial growth media; CDI: Clostridium difficile infection; CPCOA: constrained principal coordinates analysis; QIIME 2: quantitative insights into microbial ecology 2.
\end{abstract}

\section{Acknowledgements}

The authors gratefully acknowledge Zhongshan Hospital Department of Gastroenterology, Xiamen University for human intestinal microbiota samples.

\section{Authors' contributions}

F-YS designed and performed all the experiments; F-YS analyzed the 16s rRNA sequencing data; F-YS, C-KN and Z-JN performed statistical analysis. F-YS, C-KN, $Z-J N, X-C X, F-L N, Z-B Z, F-B S$ and R-JL prepared the manuscript, evaluated the results and revised the manuscript. All authors read and approved the final manuscript.

\section{Funding}

This project was supported by the National Natural Science Foundation of China (No. 81770558), and Xiamen Joint Projects for Major Diseases (No. 3502Z20149031).

\section{Availability of data and materials} Not applicable.

\section{Ethics approval and consent to participate}

The study was approved by Xiamen University and Zhongshan Hospital of Xiamen University, China. This study did not involve human study and human tissue. All fecal microbiota transplantation donors provided written informed consent. In vitro cultures were performed in compliance with the relevant laws and Xiamen University policies.

\section{Consent for publication}

Not applicable.

\section{Competing interests}

The authors declare that they have no competing interests.

Received: 15 November 2018 Accepted: 6 May 2019 Published online: 23 May 2019
References

Bang SJ, Kim G, Mi YL, Song EJ, Jung DH, Kum JS, Nam YD, Park CS, Seo DH (2018) The influence of in vitro pectin fermentation on the human fecal microbiome. Amb Express 8(1):98. https://doi.org/10.1186/s1356 8-018-0629-9

Barberan A, Bates ST, Casamayor EO, Fierer N (2012) Using network analysis to explore co-occurrence patterns in soil microbial communities. ISME J 6(2):343-351. https://doi.org/10.1038/ismej.2011.119

Brisse S, Grimont F, Grimont PAD (2006) The genus Klebsiella. In: Dworkin M, Falkow S, Rosenberg E, Schleifer K-H, Stackebrandt E (eds) The prokaryotes, vol 6. Proteobacteria: gamma subclass. Springer, New York, pp 159-196

Callahan BJ, Mcmurdie PJ, Rosen MJ, Han AW, Johnson AJA, Holmes SP (2016) DADA2: high-resolution sample inference from Illumina amplicon data. Nat Methods 13(7):581-583. https://doi.org/10.1038/nmeth.3869

Carlson JL, Erickson JM, Hess JM, Gould TJ, Slavin JL (2017) Prebiotic dietary fiber and gut health: comparing the in vitro fermentations of beta-glucan, inulin and xylooligosaccharide. Nutrients. https://doi.org/10.3390/ nu9121361

Eckburg PB, Bik EM, Bernstein CN, Purdom E, Dethlefsen L, Sargent M, Gill SR, Nelson KE, Relman DA (2005) Diversity of the human intestinal microbial flora. Science 308(5728):1635. https://doi.org/10.1126/science.1110591

Faust K, Sathirapongsasuti JF, Izard J, Segata N, Gevers D, Raes J, Huttenhower C (2012) Microbial co-occurrence relationships in the human microbiome. PLoS Comput Biol 8(7):e1002606. https://doi.org/10.1371/journ al.pcbi.1002606

Fusco A, Savio V, Cammarota M, Alfano A, Schiraldi C, Donnarumma G (2017) Beta-defensin-2 and beta-defensin-3 reduce intestinal damage caused by salmonella typhimurium modulating the expression of cytokines and enhancing the probiotic activity of Enterococcus faecium. J Immunol Res 2017:6976935. https://doi.org/10.1155/2017/6976935

Goodman AL, Kallstrom G, Faith JJ, Reyes A, Moore A, Dantas G, Gordon J (2011) Extensive personal human gut microbiota culture collections characterized and manipulated in gnotobiotic mice. Proc Natl Acad Sci USA 108(15):6252-6257. https://doi.org/10.1073/pnas.1102938108

Gupta A, Khanna S (2017) Fecal microbiota transplantation. Jama 318(1):102. https://doi.org/10.1001/jama.2017.6466

Jin W, Han K, Dong S, Yang Y, Mao Z, Su M, Zeng M (2018) Modifications in gut microbiota and fermentation metabolites in the hindgut of rats after the consumption of galactooligosaccharide glycated with fish peptide. Food Funct. https://doi.org/10.1039/C7FO02002C

Kassinen A, Krogius-Kurikka L, Makivuokko H, Rinttila T, Paulin L Corander J, Malinen E, Apajalahti J, Palva A (2007) The fecal microbiota of irritable bowel syndrome patients differs significantly from that of healthy subjects. Gastroenterology 133(1):24-33. https://doi.org/10.1053/j.gastr 0.2007 .04 .005

Kovatcheva-Datchary P, Nilsson A, Akrami R, Lee YS, De Vadder F, Arora T, Hallen A, Martens E, Bjorck I, Backhed F (2015) Dietary fiber-induced improvement in glucose metabolism is associated with increased abundance of Prevotella. Cell Metab 22(6):971-982. https://doi.org/10.1016/j. cmet.2015.10.001

Lagier JC, Dubourg G, Million M, Cadoret F, Bilen M, Fenollar F, Levasseur A, Rolain JM, Fournier PE, Raoult D (2018) Culturing the human microbiota and culturomics. Nat Rev Microbiol. https://doi.org/10.1038/s4157 9-018-0041-0

Lau JT, Whelan FJ, Herath I, Lee CH, Collins SM, Bercik P, Surette MG (2016) Capturing the diversity of the human gut microbiota through cultureenriched molecular profiling. Genome Med 8(1):72. https://doi. org/10.1186/s13073-016-0327-7

Macfarlane GT, Allison C, Gibson SA, Cummings JH (1988) Contribution of the microflora to proteolysis in the human large intestine. J Appl Microbiol 64(1):37-46. https://doi.org/10.1111/j.1365-2672.1988.tb02427.x

Makki K, Deehan EC, Walter J, Backhed F (2018) The impact of dietary fiber on gut microbiota in host health and disease. Cell Host Microbe 23(6):705715. https://doi.org/10.1016/j.chom.2018.05.012

Mcdonald JAK, Schroeter K, Fuentes S, Heikamp-Dejong I, Khursigara CM, Vos WMD, Allen-Vercoe E (2013) Evaluation of microbial community reproducibility, stability and composition in a human distal gut chemostat model. J Microbiol Methods 95(2):167-174. https://doi.org/10.1016/j. mimet.2013.08.008 
Nakano V, Ignacio A, Fernandes MR, Fugukaiti MH, Avila-Campos MJ (2013) Intestinal Bacteroides and Parabacteroides species producing antagonistic substances. Curr Trends Immunol 1:1-4

Pham VT, Mohajeri MH (2018) The application of in vitro human intestinal models on the screening and development of pre- and probiotics. Benef Microb. https://doi.org/10.3920/BM2017.0164

Quast C, Pruesse E, Yilmaz P, Gerken J, Schweer T, Yarza P, Peplies J, Glockner FO (2013) The SILVA ribosomal RNA gene database project: improved data processing and web-based tools. Nucleic Acids Res 41(Database issue):D590-D596. https://doi.org/10.1093/nar/gks1219

Reese AT, Dunn RR (2018) Drivers of microbiome biodiversity: a review of general rules, feces, and ignorance. Mbio. https://doi.org/10.1128/ mbio.01294-18

Ren YD, Ye ZS, Yang LZ, Jin LX, Wei WJ, Deng YY, Chen XX, Xiao CX, Yu XF, Xu $H Z$ (2017) Fecal microbiota transplantation induces hepatitis $B$ virus e-antigen ( $\mathrm{HBeAg}$ ) clearance in patients with positive HBeAg after longterm antiviral therapy. Hepatology 65(5):1765. https://doi.org/10.1002/ hep.29008

Routy B, Gopalakrishnan V, Daillere R, Zitvogel L, Wargo JA, Kroemer G (2018) The gut microbiota influences anticancer immunosurveillance and general health. Nat Rev Clin Oncol 15(6):382-396. https://doi.org/10.1038/ s41571-018-0006-2

Schneeberger M, Everard A, Gomez-Valades AG, Matamoros S, Ramirez S, Delzenne NM, Gomis R, Claret M, Cani PD (2015) Akkermansia muciniphila inversely correlates with the onset of inflammation, altered adipose tissue metabolism and metabolic disorders during obesity in mice. Sci Rep 5:16643. https://doi.org/10.1038/srep16643

Sekirov I, Russell SL, Antunes LC, Finlay BB (2010) Gut microbiota in health and disease. Physiol Rev 90(3):859-904. https://doi.org/10.1152/physr ev.00045.2009

Seo M, Heo J, Yoon J, Kim SY, Kang YM, Yu J, Cho S, Kim H (2017) Methanobrevibacter attenuation via probiotic intervention reduces flatulence in adult human: a non-randomised paired-design clinical trial of efficacy. PLoS ONE 12(9):e0184547. https://doi.org/10.1371/journal.pone.0184547

Soto M, Herzog C, Pacheco JA, Fujisaka S, Bullock K, Clish CB, Kahn CR (2018) Gut microbiota modulate neurobehavior through changes in brain insulin sensitivity and metabolism. Mol Psychiatry. https://doi.org/10.1038/ s41380-018-0086-5

Staley C, Kaiser T, Vaughn BP, Graiziger CT, Hamilton MJ, Rehman Tu, Song K, Khoruts A, Sadowsky MJ (2018) Predicting recurrence of Clostridium difficile infection following encapsulated fecal microbiota transplantation. Microbiome 6(1):166. https://doi.org/10.1186/s40168-018-0549-6
Surawicz CM, Brandt L, Binion DG, Ananthakrishnan AN, Curry SR, Gilligan PH, Mcfarland LV, Mark M, Zuckerbraun BS (2013) Guidelines for diagnosis, treatment, and prevention of Clostridium difficile infections. Am J Gastroenterol 108(4):478-494. https://doi.org/10.1038/ajg.2013.4

Thomson P, Medina DA, Ortuzar V, Gotteland M, Garrido D (2018) Anti-inflammatory effect of microbial consortia during the utilization of dietary polysaccharides. Food Res Int 109:14-23. https://doi.org/10.1016/j.foodr es.2018.04.008

Tramontano M, Andrejev S, Pruteanu M, Klünemann M, Kuhn M, Galardini M, Jouhten P, Zelezniak A, Zeller G, Bork P (2018) Nutritional preferences of human gut bacteria reveal their metabolic idiosyncrasies. Nature Microbiology. https://doi.org/10.1038/nature07540

Tremaroli V, Bäckhed F (2012) Functional interactions between the gut microbiota and host metabolism. Nature 71(7415):242-249. https://doi. org/10.1038/nature11552

Wang X, Wang X, Jiang H, Cai C, Li G, Hao J, Yu G (2018) Marine polysaccharides attenuate metabolic syndrome by fermentation products and altering gut microbiota: an overview. Carbohydr Polym 195:601-612. https://doi. org/10.1016/j.carbpol.2018.05.003

Williams CF, Walton GE, Jiang L, Plummer S, Garaiova I, Gibson GR (2015) Comparative analysis of intestinal tract models. Ann Rev Food Sci Technol 6(1):329-350. https://doi.org/10.1146/annurev-food-022814-015429

Yan D, Zhang T, Su J, Zhao LL, Wang H, Fang XM, Zhang YQ, Liu HY, Yu LY (2018) Structural variation in the bacterial community associated with airborne particulate matter in Beijing, China, during hazy and nonhazy days. Appl Environ Microbiol. https://doi.org/10.1128/aem.00004-18

Zadehtahmasebi M, Duca FA, Rasmussen BA, Bauer PV, Côté CD, Filippi BM, Lam TKT (2016) Activation of short and long chain fatty acid sensing machinery in the ileum lowers glucose production in vivo. J Biol Chem 291(16):8816-8882. https://doi.org/10.1074/jbc.M116.718460

Zhou W, Yan Y, Mi J, Zhang H, Lu L, Luo Q, Li X, Zeng X, Cao Y (2018) Simulated digestion and fermentation in vitro by human gut microbiota of polysaccharides from bee collected pollen of Chinese Wolfberry. J Agric Food Chem. https://doi.org/10.1021/acs.jafc.7b05546

\section{Publisher's Note}

Springer Nature remains neutral with regard to jurisdictional claims in published maps and institutional affiliations.

\section{Submit your manuscript to a SpringerOpen ${ }^{\odot}$ journal and benefit from:}

- Convenient online submission

- Rigorous peer review

- Open access: articles freely available online

- High visibility within the field

- Retaining the copyright to your article

Submit your next manuscript at $\boldsymbol{\nabla}$ springeropen.com 